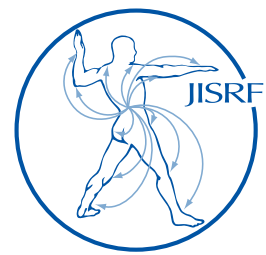

\title{
Anterolateral Oblique Distal Femoral Osteotomy for the Removal of Well Fixed Cemented Femoral TKA Components
}

Fehring $K^{1}$, Wyles $C^{2}$, Martin ${ }^{1}$, Trousdale $R^{2}$

\begin{abstract}
In the setting of periprosthetic joint infection, the complete removal of implants and cement can be challenging with well-fixed, cemented implants about the knee. This can get especially complex in the setting of long cemented femoral component stems. Osteotomies are well described in the proximal femur and tibia for removal of implants and cement. There is little information available on distal femoral osteotomies. We describe an anterolateral oblique distal femoral osteotomy for the removal of well-fixed, cemented components in resection knee arthroplasty that preserves vascularity to the osteotomized segment.
\end{abstract}

Keywords: distal femoral osteotomy; revision TKA; complex revision Level of Evidence: AAOS Therapeutic Level III

\section{Introduction}

The removal of well-fixed implants with cemented stems about the knee presents unique challenges in revision and resection knee arthroplasty. [1- $\underline{3}]$ Extended trochanteric osteotomies and tibial tubercle osteotomy for revision hip and knee arthroplasty have been previously described and used effectively for the removal of implants in these settings. [4- $\underline{4}]$ Anterior femoral osteotomy in revision knee arthroplasty has been previously described, however many of these reports do not outline the specific technique utilized or markedly compromise blood flow to the osteotomized fragment. [1,9] These techniques were utilized in small groups of patients with limited follow up.

In the setting of periprosthetic infection, all implants and cement mantle should be removed in order to eradicate infection. Removing cement in the femoral canal from
1 Keith A Fehring, MD; J. Ryan Martin, MD

OrthoCarolina, Department of Orthopaedic Surgery, 250 N Caswell Rd, Charlotte, NC 28207

(Direct reprint requests to Ryan Martin)

2 Cody C Wyles, MD; Robert T Trousdale, MD

Mayo Clinic, Department of Orthopaedic Surgery, Rochester, MN 55905

(C) 2017 Fehring, Wyles, Martin, Trousdale. All rights reserved. Authors retain copyright and grant the journal right of first publication with the work. Reconstructive Review is an open access publication and follows the Creative Commons Attribution-NonCommercial CC BY-NC. This license allows anyone to download works, build upon the material, and share them with others for noncommercial purposes as long as they credit the senior author, Reconstructive Review, and the Joint Implant Surgery \& Research Foundation (JISRF). An example credit would be: "Courtesy of (senior author's name), Reconstructive Review, JISRF, Chagrin Falls, Ohio". 
the distal femur is time-consuming, and leaves uncertainty as to whether all cement has been cleared from the femur. A femoral osteotomy can be effective in allowing direct visualization of the femoral canal facilitating the removal of implants, as well as the cement mantle during debridement. This technique is specifically useful in the setting of infection where removal of all foreign material is paramount.

We describe a technique of anterolateral oblique distal femoral osteotomy to remove well-fixed, cemented femoral implants while preserving blood supply to the lateral osteotomized fragment. We have used this technique only in the setting of periprosthetic infection with fully cemented femoral stems, however it may be applicable to other situations in revision arthroplasty.

\section{Technique}
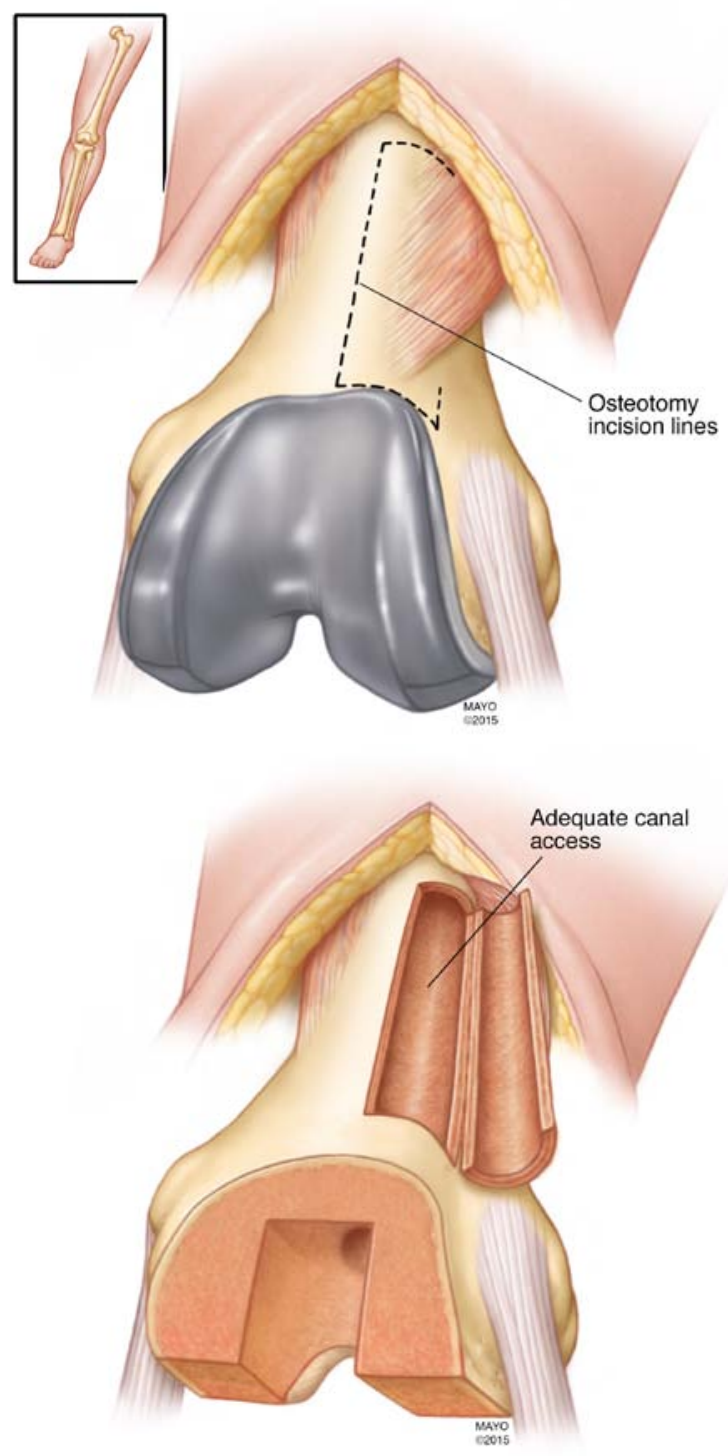

Figures 1 and 2.
A standard midline incision with a medial parapatellar arthrotomy is utilized to approach the knee. Synovectomy is performed as necessary; however care is taken not to devascularize the anterolateral aspect of the distal femur. An oscillating saw and osteotomes are used to free up the cement-implant interface along the anterior, posterior, distal, and chamfer portions of the femoral component. An impactor is used to attempt removal of the implant. If the stem is well fixed, or cement is not able to be viewed from the distal femur, then the decision to proceed with osteotomy is made. Sharp dissection is then carried out on the anterior aspect of the femur, while leaving the anterolateral and lateral soft tissue attachments preserved. Using cautery a line is made in the midline of the anterior aspect of the femur and brought out laterally at the proximal most point of the osteotomy. The length of the osteotomy will vary based on the length of the stem and cement mantle. The distal osteotomy site is made just proximal to the anterior phalange of the knee replacement. An oscillating saw is then used to perform the osteotomy entering the femur directly anterior at an oblique angle towards the posterolateral cortex. Osteotomes are then used to book open the fragment and the fragment exits posterolaterally on the femur distally ending at the lateral epicondyle and exiting posterior to the lateral intramuscular septum. Care is taken to assure the lateral and posterior soft tissue envelope is left undisturbed and that the osteotomy hinges laterally without propagating a fracture. (Figure 2) Pre-drilling of the lateral femoral cortex may further reduce the risk of fracture propagation while opening the osteotomy site. Retractors may be placed inside the osteotomized fragment to visualize the canal. Once the osteotomy is performed, direct access to the femoral canal is gained. (Figure 3) If the stemmed implant is still present, a pencil tip burr is used to go circumferentially around the stem to facilitate removal. The implant should be able to be

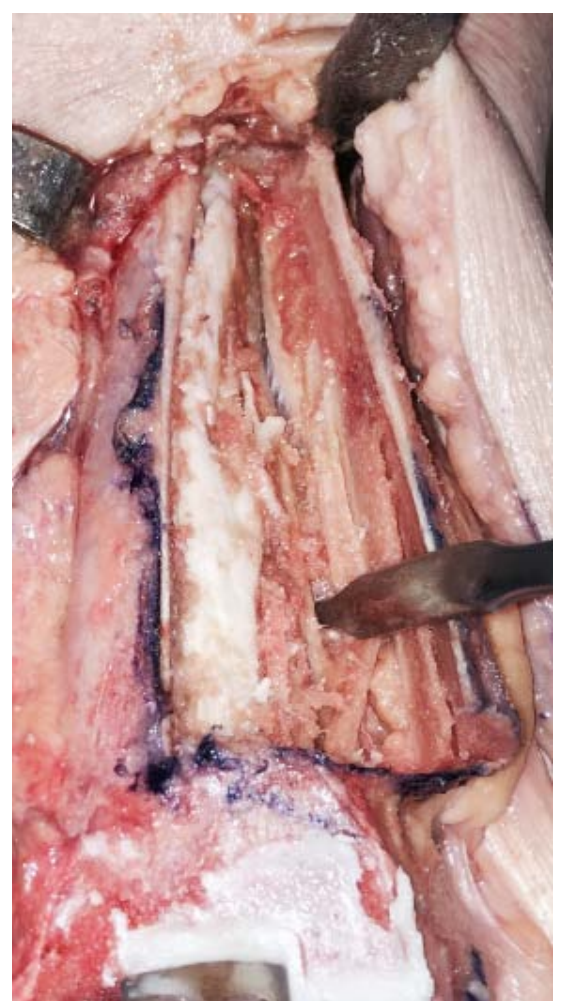

Figure 3. 


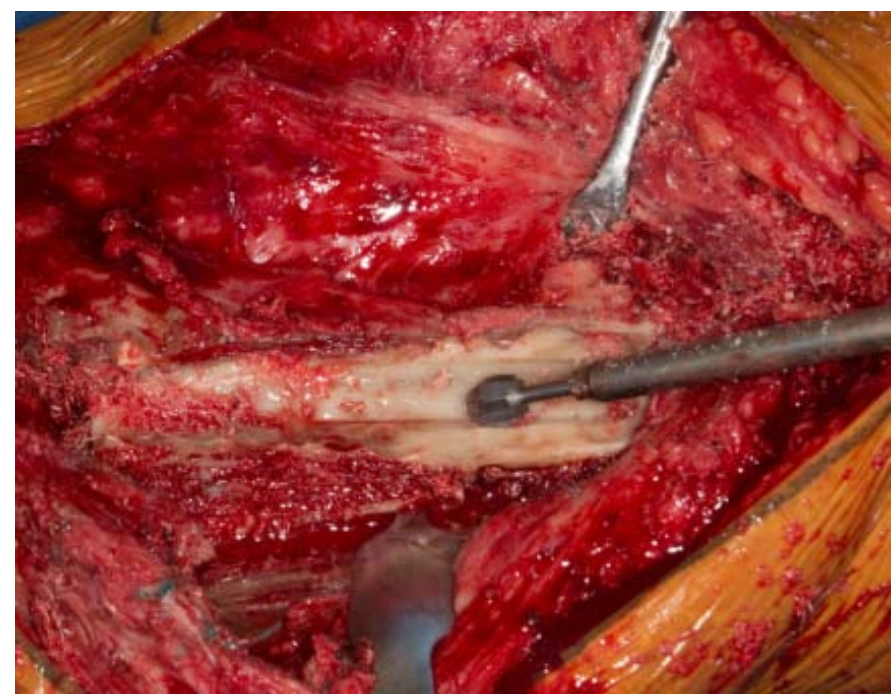

Figure 4.

removed at this time. Often after removal, there may be an abundance of cement mantle still present that must be removed. A high-speed burr is used to remove retained cement in the femoral canal and get to bleeding bone surfaces. (Figure 4) With direct visualization, one can see the cement mantle that is smoothed off that would be otherwise obscured using a distal removal technique. This technique can be performed with or without the use of a tibial tubercle osteotomy to remove the tibial component and cement mantle. Once adequate debridement is performed and all of the cement is removed, a static spacer is utilized. Two dowel rods are fashioned using plastified molds and antibiotic impregnated cement (Vancomycin 3 grams and Gentamicin 3 grams) to be inserted in the femoral and tibial canals. These dowel rods, in addition to a block of cement to support the articulation and fill the space underneath the extensor mechanism form a static
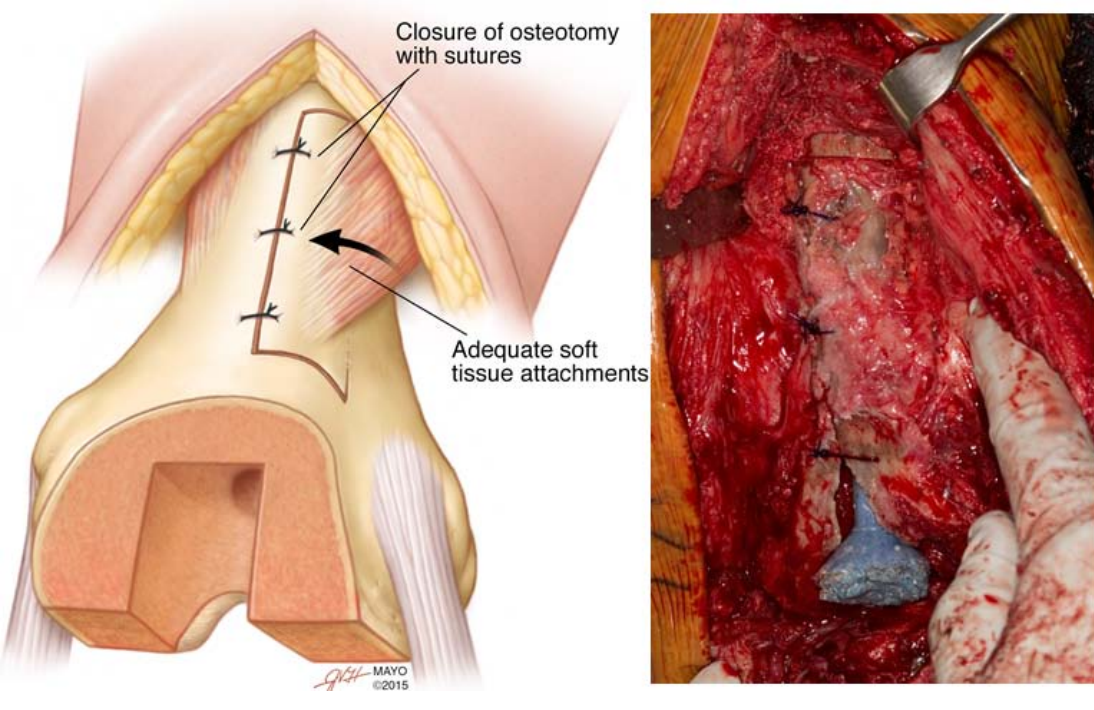
spacer for the knee. The osteotomized fragment of the femur is then reduced and fixated using large non-braided, monofilament suture through drill holes in the fragment. (Figure 5) The patient is kept toe-touch weight bearing for 8 weeks until healing of the osteotomy is present on radiographs. Most resection arthroplasty patients routinely have restricted weight bearing during the post-operative period allowing the osteotomy time to heal prior to re-implantation, thus there is no unnecessary delay. At time of reimplantation and healing of the osteotomy, a long stem is used assuring to bypass the osteotomy. Cemented or uncemented stems can be utilized at the time of reimplantation. Hybrid fixation can

Figure 5.
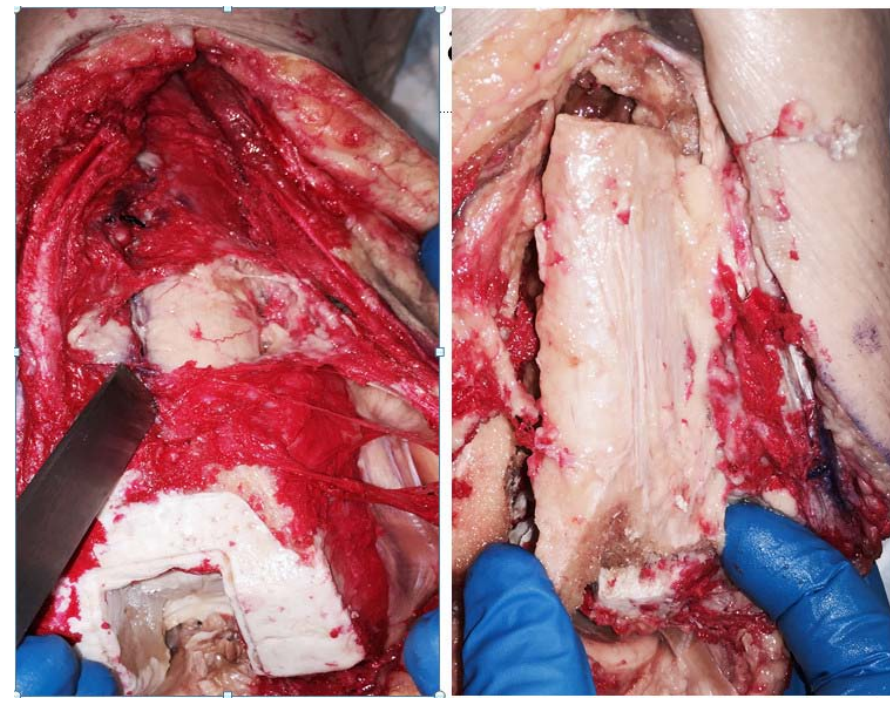

Figure 6. also be considered with uncemented metaphyseal cones and cemented stems.

\section{Patients and Methods}

We have utilized this technique at the time of resection arthroplasty for chronic periprosthetic knee infection in two patients with well-fixed, cemented femoral stems. A comparative qualitative analysis of blood supply to the distal femur was also performed using four fresh-frozen cadaveric knees injected with latex following this anterolateral osteotomy in right and left specimens, an anterior based osteotomy, as well as a control specimen where no osteotomy was performed. Each osteotomy was performed on the cadaveric specimens. The femoral artery was then cannulated and injected with latex dye. We then froze the specimens to allow procurement of the latex. The speci- 
mens were then analyzed for blood supply to the distal femur and the osteotomized fragment.

\section{Results}

Both patients showed healing of the osteotomy at the time of reimplantation and were successfully reconstructed utilizing stems bypassing the site of the osteotomy. There were no complications using this technique. Both patients were reimplanted using a constrained hinge knee prosthesis. Both are infection free and weight bearing as tolerated at latest follow up. Comparative qualitative analysis of blood supply to the distal femur following the different osteotomy types showed a more robust blood supply when utilizing the anterolateral osteotomy compared to the anterior based osteotomy. (Figure 6) The blood supply to the distal femur in the specimens with the anterolateral osteotomy was similar to the control specimen where no osteotomy was performed.

\section{Discussion}

Osteotomy of the femur is often unnecessary in the removal of well-fixed femoral implants, and should be reserved for special circumstances due to its risk of nonunion, fracture propagation, and compromised fixation at revision knee arthroplasty. We present a technique that can be effectively utilized to remove well-fixed femoral components and the cement mantle in resection arthroplasty for periprosthetic knee infection.

One recent report describes an anterior osteotomy entering medially and exiting laterally on the anterior portion of the femur. [9] There are several flaws and differences in this type of osteotomy performed for the removal of wellfixed components. This described osteotomy threatens the vascularity of the osteotomized fragment as this technique makes it difficult to preserve the lateral soft tissue attachments to the anterior based fragment. This is especially disadvantageous in the setting of infection. Previous reports showing the subperiosteal blood supply to the distal femur highlight the lateral cortex and condyle as containing an abundance of intraosseous and extraosseous blood supply. $[10,11]$ The anterior femur is vascularized by branches of the superior medial and lateral geniculate arteries in addition to the anterior soft tissues. An anterior based fragment will compromise this feeding vasculature from the medial and lateral sides of the femur. Furthermore, more soft tissue stripping is needed to complete this type of osteotomy. Our described surgical technique leaves the lateral soft tis- sue sleeve undisturbed. (Figure 3-3)

The anterior osteotomy described by Merz et al. was fixed using circumferential cables around the distal femur bringing the unnecessary threat of vascular complications to this procedure. Further periosteal stripping around the osteotomy must be performed to properly pass circumferential cables safely which may threaten viability to the osteotomized fragment.

Our technique of large caliber suture fixation has the advantage of superior visualization, adequate stabilization, fragment viability, and is theoretically safer than the previously described technique of osteotomy fixation. (Figure 2-2 and Figure 3-3) We have had no intra-operative complications using this technique. We have found this osteotomy to be a useful technique when removing well-fixed, cemented femoral components during periprosthetic infection.

To date, we have utilized this technique at the time of resection arthroplasty for periprosthetic infection in two patients with well-fixed, cemented femoral stems. Both patients showed healing of the osteotomy at the time of reimplantation and were successfully reconstructed utilizing stems bypassing the site of the osteotomy. Both patients are infection free and weight bearing as tolerated at 2-year follow-up.

\section{Disclosure}

The authors declare that there is no conflict of interest regarding the publication of this paper. For full disclosures refer to last page of this journal.

\section{References}

1. Massin P, Boyer P, Sabourin M, Jeanrot C. Removal of infected cemented hinge knee prostheses using extended femoral and tibial osteotomies: Six cases. Orthop Traumatol Surg Res. 2012;98(7):840-844.

2. Masri BA, Mitchell PA, Duncan CP. Removal of solidly fixed implants during revision hip and knee arthroplasty. J Am Acad Orthop Surg. 2005;13(1):18-27.

3. Mason JB, Fehring TK. Removing well-fixed total knee arthroplasty implants. Clin Orthop Relat Res. 2006;446:76-82.

4. Glassman AH, Engh CA, Bobyn JD. A technique of extensile exposure for total hip arthroplasty. J Arthroplasty. 1987;2(1):11-21.

5. Glassman AH. Exposure for revision: Total hip replacement. Clin Orthop Relat Res. 2004;(420)(420):39-47.

6. Archibeck MJ, Rosenberg AG, Berger RA, Silverton CD. Trochanteric osteotomy and fixation during total hip arthroplasty. J Am Acad Orthop Surg. 2003;11(3):163173.

7. Whiteside LA, Ohl MD. Tibial tubercle osteotomy for exposure of the difficult total knee arthroplasty. Clin Orthop Relat Res. 1990;(260)(260):6-9.

8. Whiteside LA. Exposure in difficult total knee arthroplasty using tibial tubercle osteotomy. Clin Orthop Relat Res. 1995;(321)(321):32-35.

9. Merz MK, Farid YR. Anterior distal femoral osteotomy for removal of long femoral stems in revision knee arthroplasty. J Arthroplasty. 2014;29(7):1423-1425.

10. Penteado CV, Masquelet AC, Romana MC, Chevrel JP. Periosteal flaps: Anatomical bases of sites of elevation. Surg Radiol Anat. 1990;12(1):3-7.

11. ROGERS WM, GLADSTONE H. Vascular foramina and arterial supply of the distal end of the femur. J Bone Joint Surg Am. 1950;32(A:4):867-874. 